\title{
Socio-Anthropologie Du Processus D'autonomisation Des Femmes Rurales Ivoiriennes À Travers Les Activités Génératrices De Revenus
}

\author{
Youl Félix \\ Kouame Aya Lydie-Marcelle \\ Kouakou Aya Larissa \\ Toh Alain \\ Laboratoire d'Etudes et de Recherches Interdisciplinaires \\ en Sciences Sociales Institut d'Ethno-Sociologie \\ Université Félix Houphouët-Boigny d'Abidjan, Côte d'Ivoire
}

Doi: 10.19044/esj.2017.v13n32p303 URL:http://dx.doi.org/10.19044/esj.2017.v13n32p303

\begin{abstract}
This article focuses on the rationale behind women's choices of economic activities in the Gomon Sub-Prefecture and the methods used to implement these activities. Specifically, it describes the strategies used by the women of the Gomon Sub-prefecture to ensure their financial autonomy and provides insights into the difficulties behind syndicating these women. Using both qualitative and quantitative assessment tools, this study targeted women involved in income-generating activities in the sub-prefecture. To overcome poverty, women have invested in the production, processing and marketing of food. The decision-making process described in this article demonstrates that these women make rational choices in the daily management of their incomegenerating activities aimed specifically at maximizing the economic gains necessary to their financial autonomy and the improvement of their social status.
\end{abstract}

Keywords: Rural women, socio-economic activity, rationale, empowerment, poverty

\section{Resume}

Le présent article porte sur les logiques qui président au choix des activités économiques des femmes de la Sous-préfecture de Gomon et aux modes de pratiques de leurs activités. De manière spécifique, il met en relief les stratégies mises en œuvre par les femmes de la Sous-préfecture de Gomon pour garantir leur autonomie financière ainsi que les facteurs explicatifs de la 
difficulté de regroupement de ces femmes. Dans une approche qualitative et quantitative, cette étude à questionner principalement les femmes qui exercent des activités génératrices de revenu dans la sous-préfecture. Dans 1'objectif de s'affranchir de la pauvreté, elles sont investies dans la production, la transformation et la commercialisation du vivrier. La théorie de la décision retenue pour ce travail met en évidence les choix rationnels opérés par ces femmes dans la pratique de leurs activités en vue de maximiser leur gain économique, condition de leur autonomisation financière et de valorisation de leur statut social.

Mots-clés: Femme rurale, activité socio-économique, logiques,
autonomisation, pauvreté

\section{Introduction}

L'élimination de l'extrême pauvreté demeure l'un des plus grand défis de notre temps et constitue l'une des principales préoccupations de la communauté internationale. La question de la pauvreté de la femme dans ce combat retient particulièrement 1'attention des décideurs politiques. Selon la Banque Mondiale, $75 \%$ des personnes vivant dans les conditions de pauvreté extrême se trouvent dans les zones rurales. Les femmes sont, par ailleurs, les plus vulnérables à cette pauvreté (Banque Mondiale, 2004). Les objectifs du millénaire pour le développement en leur troisième objectif consacrent cette lutte et indiquent la voie à suivre pour lutter contre la pauvreté de la femme. Il faut promouvoir l'égalité des sexes et l'autonomisation des femmes pour les aider à sortir de leur précarité. Elles sont ainsi, encouragées à faire preuve de créativité et à entreprendre des actions concrètes et innovantes qui peuvent les aider à s'affranchir de la pauvreté. Michèle Bachelet a lancé un message aux femmes à ce sujet, à l'occasion de la journée internationale de la femme 2012. La directrice exécutive de l'ONU Femme ${ }^{57}$, s'adressant aux femmes du monde, notamment à celles qui vivent en milieu rural, les a invitées à se rendre autonome pour ne pas rester en marge de la lutte contre la misère et à contribuer à l'éradication de la faim et de la pauvreté dans le monde.

Les femmes rurales, responsables de la moitié de la production mondiale alimentaire, d'environ $80 \%$ de la majorité de la production alimentaire dans les pays en voie de développement, ne perçoivent que $1 \%$ des revenus et ne possèdent que $1 \%$ des terres agricoles (FAO, 2010). En Côte d'Ivoire, l'organisation sociale traditionnelle a laissé à la femme la charge de garantir totalement la subsistance familiale. Ainsi, l'agriculture vivrière et la sécurité alimentaire ont toujours relevé de sa responsabilité (Baha $\mathrm{Bi}, 2001$ ). Le secteur vivrier détenu par celles-ci a généralement été perçu comme un

\footnotetext{
${ }^{57}$ Organisation des Nations Unis pour la femme
} 
secteur accessoire de l'économie nationale d'où le peu d'intérêt porté à son endroit. La place de choix est dévolu à l'agriculture d'exportation avec à sa tête le binôme café-cacao. Cette option a davantage renforcé les projets de développement agricole en faveur des cultures d'exportation au détriment des cultures vivrières. Pour preuve, toutes les opérations de vulgarisations conduites par les sociétés d'encadrement s'adressent prioritairement aux chefs d'exploitations agricoles familiales, qui sont constitués à plus de 95\%, d'hommes (Vanga, 2012).

$\mathrm{Ce}$ choix politique et économique a eu pour corollaire «une féminisation de la pauvreté dans le monde paysan » (Baha $\mathrm{Bi}$, op.cit). Des inégalités structurelles ont de plus renforcé les disparités entre les hommes et les femmes. En milieu rural, les droits fonciers pour les femmes sont inexistants et la possibilité offerte à celles-ci par les législations (code civil et code foncier rural) d'avoir droit à la propriété foncière est restée très théorique. L'allocation des terres aux femmes est constamment entravée par les coutumes et mœurs traditionnelles en vigueur dans les communautés rurales (Koné, 2003).

En dépit de ces contraintes financières et institutionnelles que peuvent constituer le faible financement de l'agriculture vivrière dans lequel les femmes évoluent et l'inexistence de droits sur la terre, les femmes vont redéfinir leurs objectifs de production. Celles qui au départ pratiquaient une agriculture de subsistance familiale sur de petites parcelles de production vont s'ériger en véritables acteurs économiques. Leurs activités économiques dans le secteur du vivrier se sont généralisées dans le pays et dans ce domaine, ce sont les femmes malinké et gouro de Côte d'Ivoire qui ont acquis une notoriété (N'guessan, 1993). Ces femmes ont développé un esprit coopératif et sont initiatrices et responsables des plus grandes coopératives qui exercent dans le secteur vivrier en Côte d'Ivoire. Conscientes de leur condition sociale et économique, ces femmes ont adopté la création de coopératives comme stratégies pour contourner les contraintes qui les maintiennent dans la pauvreté (Séhi Bi, 2012 ; Vanga, op. cit).

Le présent article expose la situation des femmes de la sous-préfecture de Gomon, située dans le sud de la Côte d'Ivoire. A l'instar des femmes malinké et gouro qui exercent dans le domaine du vivrier, la population féminine de la sous-préfecture de Gomon s'est résolument engagée dans la pratique d'activités socio-économiques en rapport avec le vivrier. Il questionne les logiques sociales qui président au choix des activités économiques des femmes de la Sous-préfecture de Gomon, leurs modes et pratiques sans oublier la portée sociale de leurs activités. 


\section{Methodologie}

La recherche a été menée à partir de la théorie de la décision qui s'inscrit dans le paradigme interactionniste. La méthode dialectique a été également privilégiée pour rendre compte de la réalité dans cette étude. Les interactionnistes proposent d'analyser le social par les actions individuelles à partir de l'hypothèse selon laquelle les individus sont des sujets conscients. Ce paradigme postule que toute décision résulte de choix, de classements, des conséquences anticipées, des diverses actions possibles. Le milieu social étant pour l'acteur un point de référence à partir duquel il s'efforce de mesurer les avantages, les inconvénients ou les risques de décisions. Notre préférence également pour l'approche dialectique, part du fait qu'elle permet d'appréhender les totalités changeantes dans la société rurale de Gomon à travers le dynamisme avéré des femmes dans la pratique de leurs activités agricoles. Elle prend en compte les contradictions, le mouvement, les interactions et le changement à travers ses différentes lois :

La loi de la contradiction prend en compte le fait que les femmes disent vouloir s'affranchir de la pauvreté mais refusent de s'adonner à tous types de cultures même prisées sur les marchés. Elle prend aussi en compte leur réticence à l'idée de se constituer en coopérative en dépit du fait que les coopératives soient prises comme des préalables à l'essor de leurs activités. Elle prend aussi en compte la présence des femmes dans la production de palmier à huile connu comme une culture industrielle et non vivrière. La production de cette culture nécessite la disponibilité de la terre, eu égard à sa durée d'exploitation.

La loi du mouvement, permet de montrer l'évolution de l'activité des femmes, de faire la sociohistoire des activités rurales féminines dans cette localité. La loi de l'interaction nous permet de montrer comment les interactions existantes au sein de la communauté religieuse des Assemblées de Dieu ont fini par les socialiser et créer une identité collective favorable à leur regroupement. Enfin la loi du bond qualitatif permet de montrer la portée économique et sociale de l'activité économique des femmes.

L'enquête s'est déroulée sur une période d'un mois(1). Elle a ciblé essentiellement les femmes engagées dans la production vivrière, la transformation du manioc et la commercialisation de vivriers. Elle a été réalisée sur un échantillon de 43 femmes à qui des questionnaires ont été administrés. Ces femmes sont issues du GFPVG ${ }^{58}$ (18), du GFAG ${ }^{59}$ (7) et 18 femmes exerçants librement leurs activités. Un échantillon aléatoire stratifié a consisté à choisir dans chaque strate que constituaient le GFPVG, le GFAG et l'ensemble des femmes exerçants individuellement leurs activités, un nombre

\footnotetext{
${ }^{58}$ Groupement des Femmes Productrices de Vivriers de Gomon

${ }^{59}$ Groupement de Femmes Agricultrices de Gomon
} 
aléatoire de femmes. La sélection s'est faite en fonction des femmes présentes lors des différents passages sur le terrain. Des entretiens sémi-directifs ont été réalisés avec le bureau des femmes du GFPVG et du GFAG, le guide religieux du GFPVG et du GFAG, les autorités villageoises, l'agent de l'ANADER ${ }^{60}$, et quelques-uns des époux des femmes ciblées par l'enquête. Ces entretiens ont fait l'objet de triangulation et de validation internes.

Les informations recueillies ont permis de comprendre et d'expliquer la logique qui préside au choix des activités des femmes de la Sous-préfecture de Gomon et aux modes de pratique individuels et collectifs de leurs activités. Elles renseignent particulièrement sur les facteurs explicatifs de la difficulté de regroupement et du maintien des modes de pratique individuels et collectifs des activités socio-économiques féminines à Gomon. Les stratégies mises en œuvre par les femmes pour maximiser leur profit et la portée économique et sociale de l'activité des femmes de cette Sous-préfecture ont été aussi connues à travers les différents entretiens réalisés.

\section{Resultats}

\section{Strategies des femmes dans la lutte contre la pauvrete}

Pour tirer un profit substantiel des opportunités marchandes qui s'offrent à elles, les femmes de la Sous-préfecture de Gomon s'adonnent de façon simultanée à la production de vivriers, à la transformation du manioc en attiék $^{61}$ et à la commercialisation de ces produits. Elles sont très sélectives dans le type de vivriers à produire. Cela relève d'une stratégie qui vise à maximiser leur profit. Ces femmes opèrent des choix rationnels en fonction de la ligne d'action qui est la plus susceptible de maximiser leur récompense globale.

\section{Le type de cultures dans les activités de production comme stratégie économique de lutte contre la pauvreté}

Ce tableau met en évidence les cultures vivrières sélectionnées et produites par les femmes de la Sous-préfecture de Gomon dans l'exercice de leurs activités économiques.

${ }^{60}$ Agence Nationale d'Appui au Développement Rural

${ }^{61}$ Mets culinaires à base de couscous de manioc 
Tableau $1:$ Le type de vivriers cultivés

\begin{tabular}{|c|c||c|}
\cline { 2 - 3 } \multicolumn{1}{c|}{} & EFFECTIFS & FREQUENCES \% \\
\hline Manioc & 10 & 23,26 \\
\hline Banane & 1 & 2,33 \\
\hline Légumes & 2 & 4,65 \\
\hline Manioc et Banane & 5 & 11,63 \\
\hline Manioc, Banane et Légumes & 21 & 48,84 \\
\hline Manioc et Légumes & 1 & 2,33 \\
\hline Banane et Légumes & 3 & 6,98 \\
\hline \hline TOTAL & $\mathbf{4 3}$ & $\mathbf{1 0 0 , 0 0}$ \\
\hline
\end{tabular}

Source: Données d'enquête (Sikensi-Gomon, 2015)

Les produits vivriers sont des denrées périssables, difficiles à conserver surtout dans les ménages ruraux. Pour cela, les femmes opèrent un choix rationnel parmi les cultures à produire dans le but de les écouler rapidement sur le marché sous une forme brut, ou les proposer à la consommation sous d'autres formes. Il en est de la stratégie des femmes de faire le choix de la production du manioc, de la banane plantain et des légumes avec un pourcentage plus élevé, soit $86,06 \%$ pour la culture du manioc.

\section{- Manioc}

L'enquête menée auprès des femmes a révélé que le manioc est une culture qui leur donne pleine satisfaction. Il réussit bien dans la région, n'a pas trop d'exigence et pousse sur tout type de sols. Il se consomme des feuilles aux tubercules et sous plusieurs formes (attiéké, atoukpou, placali, cogodé, bochtiké). Elles cultivent les variétés de manioc appelées «Bingerville» et «Bonoua » classées dans la catégorie des maniocs doux, avec un temps de production de 18 mois. Leur préférence porte sur la variété dénommée "6mois" dont la durée est de 6 à 7 mois. Il peut être utilisé dans la préparation de plusieurs mets. Si la production n'est pas orientée, les femmes pourraient être contraintes de la céder en dessous de sa valeur marchande initiale. (Raffaillac, 1997).

- La banane plantain

La banane plantain sert aussi à la préparation de plusieurs mets culinaires tels que le foufou et le foutou. Elle peut être réutilisée quand elle devient trop mûre pour préparer ' $^{\prime}$ alloco ${ }^{62}$ et le claklo $^{63}$. La culture de la banane plantain permet donc aux femmes de minimiser les risques de perte et de rentabiliser en proposant leurs produits à la consommation sous diverses formes. Ces femmes produisent aussi la banane plantain à contre saison. La disponibilité de leurs produits en période de pénurie leur rapporte un plus

\footnotetext{
${ }^{62}$ Fritures de banane plantain

${ }^{63}$ Galettes faites à base de purée de banane plantain
} 
grand profit. Les femmes anticipent les diverses actions possibles et retiennent celles qui leur permettent de tirer un meilleur avantage des opportunités marchandes.

- $\quad$ Légumes (Gombo, aubergine, ...)

Le gombo est un légume dont la culture nécessite peu d'attention. Il met trois mois pour germer et la récolte peut se faire plusieurs fois sur une période de quatre mois. Il est de ce fait approprié pour les activités économiques, selon les femmes. Il se consomme sous plusieurs formes également. A partir du gombo frais les femmes peuvent obtenir un autre produit appelé "Djoumgblé" qui est le gombo séché et moulu. L'aubergine comme le gombo sert aussi à la préparation de plusieurs repas.

L'agent de l'Agence Nationale d'Appui au Développement Rural (ANADER) nous confiait que la terre de la sous-préfecture de Gomon est propice à la culture de plusieurs produits maraîchers tels que la tomate, le choux, etc... Il a incité les femmes à entreprendre ces cultures mais sans succès. Le GFPVG a entrepris la culture de la tomate avec un résultat stupéfiant. Les femmes se sont cependant détournées de cette culture pour ne pas enregistrer de perte selon elles, car il est difficile à conserver. Leur logique est de maximiser leur gain économique en minimisant les risques de perte.

Tableau 1 : Niveau de production par récolte en fonction de l'âge

\begin{tabular}{|c|c|c|c|c|c|c|c|c|c|c|}
\hline \multirow{2}{*}{$\begin{array}{l}\text { Ages } \\
\text { Prodtion } \\
\text { par récolt }\end{array}$} & \multicolumn{2}{|c|}{ [25-35[ } & \multicolumn{2}{|c|}{ [35-45[ } & \multicolumn{2}{|c|}{ [45-55[ } & \multicolumn{2}{|c|}{ [55 ans et plus } & \multicolumn{2}{|c|}{ Total } \\
\hline & Eff & Fréq(\%) & Eff & Fréq(\%) & Eff & Fréq(\%) & Eff & Fréq(\%) & Eff & Fréq(\%) \\
\hline $\begin{array}{l}{[1-5 \text { sacs de }} \\
50 \mathrm{~kg} \mathrm{[}\end{array}$ & 1 & 2,33 & 3 & 6,98 & 1 & 2,33 & 1 & 2,33 & 6 & 13,95 \\
\hline $\begin{array}{l}{[5-10 \mathrm{sacs}} \\
\mathrm{de} 50 \mathrm{~kg}[\end{array}$ & 2 & 4,65 & 3 & 6,98 & 0 & - & 1 & 2,33 & 6 & 13,95 \\
\hline $\begin{array}{l}{[10-15 \text { sacs }} \\
\text { de } 50 \mathrm{~kg}[\end{array}$ & 1 & 2,33 & 1 & 2,33 & 1 & 2,33 & 0 & - & 3 & 6,98 \\
\hline $1 / 2$ tonne & 0 & - & 1 & 2,33 & 1 & - & 2 & 4,65 & 3 & 6,98 \\
\hline 1,5 tonnes & 0 & - & 1 & 2,33 & 0 & 23,26 & 7 & 16,28 & 18 & 41,86 \\
\hline 2 tonnes & 0 & - & 4 & 9,30 & 10 & 6,98 & 0 & 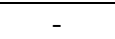 & 7 & 16,28 \\
\hline Total & 4 & 9,30 & 13 & 30,23 & 3 & 34,88 & 11 & 25,58 & 43 & 100,00 \\
\hline
\end{tabular}

Source : Données d'enquête (Sikensi-Gomon 2015)

Ces données montrent que la moitié des femmes, soit $58,14 \%$ produisent entre 1,5 tonnes et 2 tonnes. Ces femmes appartiennent aux catégories d'âge allant de 35 à 55 ans et plus. Les femmes sont beaucoup plus actives dans la production au-delà de 35 ans. A cet âge, en milieu rural, les femmes ont pour la plupart déjà eu leur dernière maternité et peuvent se consacrer aisément à la pratique de leurs activités. Les charges au niveau familial augmentent (scolarisation des enfants, habillement, soins de santé, etc.) et pour subvenir aux besoins de leur famille, les femmes se consacrent davantage à leurs activités. L'on estime aussi à cet âge, qu'il est indéniable d'avoir une réalisation personnelle (maison, cuisine, ...) et de participer à la 
vie sociale (funérailles, mariage, et autre cotisation). "La société vous juge sévèrement quand vous n'êtes pas à même de participer économiquement à la vie de la communauté, à un certain âge » tels sont les propos tenus par la majorité des femmes. Cela signifie qu'à un plus jeune âge, la société est plus tolérante quand vous n'êtes pas à mesure de participer à la vie sociale mais vous blâme quand à un âge mûr vous ne participez pas à la vie sociale. Cela est d'autant plus grave que madame B.A., organisatrice en chef du GFPVG nous confie: «J'ai utilisé toutes mes économies pour aider à l'organisation de funérailles de mes parents ».

La crainte du rejet et du blâme ainsi que la recherche d'une estime sociale incitent les femmes de la tranche d'âge [35;45[, [45 ; 55[, [55ans et plus [à s'impliquer davantage que les plus jeunes, dans leurs activités.

\section{Conversion du manioc en attiéke comme stratégie économique de lutte contre la pauvreté}

La transformation du manioc en attiéké est venue du peuple Adioukrou dans la sous-préfecture de Gomon. Les jeunes filles envoyées comme aideménagères en période de famine, ont ramené cette activité appréciée par les femmes Abidji comme une activité capable de générer un profit. La secrétaire du GFAG nous le confirme bien par ces propos: "Nous voulons fuir la pauvreté. Il faut alors faire ce qui est rentable. C'est pourquoi nous fabriquons aussi l'attiéké ». Et elle ajoute "il faut faire ce qui peut nous amener à nous éloigner le plus possible du manque ou de l'insuffisance des ressources financières ". Ces propos, montrent clairement que la fabrication de l'attiéké, génère un revenu satisfaisant, capable d'aider les femmes à sortir de la pauvreté. La transformation du manioc répond à un besoin d'accroissement du revenu. Les femmes poursuivent des fins stables en adaptant leurs actions en fonction de circonstances nouvelles et d'informations changeantes.

La stratégie adoptée par les femmes à travers la fabrication de l'attiéké répond au besoin de l'acquisition d'un gain maximum car c'est une activité rentable. Cette activité permet aussi de contourner la difficulté de conservation du manioc en tant que denrée périssable. En effet, Face au risque de voir la production de manioc brut perdre sa valeur intrinsèque et d'être vendu en deçà de sa valeur marchande initiale, la transformation du manioc est une réponse à la gestion de l'incertitude. Une femme de nationalité burkinabé affirmait lors de notre entretien: "J'ai assisté impuissante à la putréfaction de ma production de manioc parce que je ne savais pas faire de l'attiéké. Cela m'a incité à apprendre à faire de l'attiéké ».Cela justifie le fait que face au risque que relève la disponibilité de marchés permanents pour l'écoulement de leurs produits bruts, la transformation du manioc en attiéké soit combinée aux activités de production vivrière. C'est pour cette raison que les femmes penchent davantage pour la production du manioc. Leurs activités de 
transformation sont régulières et constituent une de leurs principales sources de revenu. C'est en cela qu'elles soutiennent que la transformation du manioc en attiéké leur procure plus de satisfaction, au niveau des retombés financières que la vente du manioc brut. La transformation du manioc en attiéké est à l'avantage des femmes qui s'y adonnent, car elles fournissent elles-mêmes la matière première nécessaire et n'ont pas de contraintes quant à la variété de manioc à choisir. Elles peuvent utiliser toutes les variétés de manioc pour la fabrication de l'atttiéké. Elles arrivent aussi, grâce à la fabrication de l'attiéké à assurer une substance à leurs familles même en période de famine. Selon la fréquence de l'activité leur bénéfice varie entre 100000 F CFA à $150000 \mathrm{~F}$ CFA par mois. Ce gain n'est pas statique, lorsque le marché est «bon », les femmes peuvent aller au-delà de ces bénéfices. A contrario lorsque le marché n'est pas «agréable » ces chiffres précités peuvent chuter. L'activité des femmes fonctionne selon l'offre et la demande.

\section{Commercialisation du vivrier comme stratégie économique de lutte contre la pauvreté}

La commercialisation du vivrier est un phénomène nouveau pour les femmes Abidji de la Sous-préfecture de Gomon. Elles n'osaient pas s'adonner à la pratique de cette activité parce que l'activité commerciale était mal appréciée par leurs époux. Ceux-ci craignaient de perdre leurs épouses et percevait plutôt l'activité commerciale comme une occupation susceptible de les exposer au vagabondage sexuel. Les échanges marchands ont pris de l'ampleur dans cette société grâce à l'arrivée des populations Malinké à vocation commerçante. Cela a favorisé l'insertion de des femmes Abidji dans le tissu commercial. Elles ne prennent pas le risque de produire sans faire ellesmêmes la commercialisation dans le but de tirer meilleur profit de leurs activités. Les propos de la secrétaire à l'organisation du GFPVG vont dans ce sens : " mon rôle est d'organiser la commercialisation de nos produits, de chercher de nouveaux marchés un peu partout dans le département et même dans les grandes villes du pays». Les femmes ont fait de ces activités de commercialisation des activités susceptibles de leur permettre de maximiser leur gain et de faire des profits. La présidente du GFAG nous rapporte que: " le peuple Abidji n'est pas un peuple consommateur de l'attiéké, c'est plus dans un souci de commercialisation que nous fabriquons l'attiéké ».

Ces femmes privilégient les activités de commercialisation, car dans une économie libérale comme celle dans laquelle nous évoluons, l'échange domine la production. Celui qui produit ne gagne pas plus que celui qui vend. Ces femmes adoptent cette stratégie pour mieux bénéficier des avantages de leurs activités de production et de transformation. Elles se rendent ainsi, avec leurs produits sur les marchés locaux, c'est-à-dire de Gomon et de Sikensi, ou plus loin à Abidjan pour écouler leurs produits. Dans leur stratégie, les femmes 
ne dissocient pas la production de la transformation du manioc et de la commercialisation de vivriers. C'est dans le souci de répondre aux objectifs de départ qu'elles se sont fixées pour lutter contre la pauvreté.

Le mode de pratique que les femmes adoptent dans la conduite de leurs activités est une autre stratégie à travers laquelle elles trouvent le moyen de rentabiliser. Les femmes organisent la production, la transformation et la commercialisation de leurs produits selon la forme qui leur convient le mieux et qu'elles jugent susceptible de leur garantir un profit. Ainsi on distingue les femmes qui exercent leurs activités de façon groupée (groupement) et les femmes qui exercent leurs activités de façon individuelle.

Au-delà de cet aspect qui relève d'une stratégie, il convient de s'intéresser à ce qui explique la difficulté de regroupement pour ces femmes et le maintien des modes de pratique individuels et collectifs, dans cette Souspréfecture.

\section{Pratique des activites generatrices de revenus des femmes}

Selon le chef du village de Gomon, le regroupement a commencé avec les planteurs de café et de cacao. Ceux-ci se regroupaient pour résoudre la difficulté liée au besoin de main d'œuvre. C'était un regroupement dans lequel ils n'avaient pas de plantations en commun. Ils travaillaient ensemble à tour de rôle dans les plantations de chacun des membres. Cependant, certains, par mauvaise foi, craignant de voir le statut social des autres s'améliorer par rapport au leur boycottaient les rassemblements. Aujourd'hui encore, il n'est pas facile de regrouper les femmes pour des raisons similaires mais aussi pour des raisons politiques. Un conflit politique existe à propos de la chefferie dans lequel les hommes sont très impliqués. Les femmes étant très attachées à leurs époux transposent ce conflit dans leur rapport, d'où la difficulté de s'unir aux autres femmes pour entreprendre des activités. Cela constitue un frein à leur regroupement. Toutefois, nous constatons qu'en dépit de ces obstacles, certaines femmes en nombre restreint parviennent à se regrouper pour mener leurs activités de production, de transformation et de commercialisation. Les groupements de femmes qui existent dans le village de Gomon sont issus de l'Eglise des Assemblées de Dieu. Ces groupements sont les premiers du genre dans le village et les seuls en activité. Les autres groupements qui ont essayé de se constituer ont enregistré un échec avéré. Il convient donc de mettre en exergue les phénomènes d'interactions sociales partant des rapports de communication (habitude des personnes dans la vie quotidienne et leur relation de proximité) qui sont d'une part à l'origine des conventions sociales. Il importe de s'intéresser pour cela aux relations sociales qui lient ces femmes entre elles et à la façon dont elles se socialisent, mais aussi à la façon dont cette socialisation forme une identité collective. 
La relation sociale est perçue comme un échange entre des individus qui n'est à leurs yeux ni arbitraire (il est orienté) ni absurde (il a une signification). Le fait de fréquenter la même communauté religieuse a placé les femmes dans un champ social. Les rapports sociaux qu'elles tissent en se côtoyant ont engendré une solidarité sociale au nom de laquelle elles se doivent de se soutenir et de s'entraider. A la question de savoir ce que représente le groupement pour elles, la plupart ont donné pour réponse: " C'est une famille où l'on s'entraide, c'est le moyen de se soutenir ». Pour d'autres, "c'est la communion fraternelle ». Elles utilisent le terme " sœur en Christ" pour se désigner et pour s'identifier. Ces femmes transposent les valeurs et les principes que leur enseigne l'église dans leurs groupements. Les rapports sociaux existants entre elles, s'affermissent davantage dans des concepts tels que " la communion fraternelle" et le groupement est pour elles le lieu d'une communion fraternelle. Elles appartiennent également, à la même chorale et sont toutes, membres de 1'A.O.C ${ }^{64}$. Leurs groupements constituent une plateforme d'échange, de communion et de raffermissement des liens de fraternité. L'idéologie de l'église a été transposée dans les groupements et a créé à la base les conditions de regroupement et d'existence du groupement. Cette idéologie est le fruit d'une socialisation faite par l'église. Monsieur K.S., membre du conseil de l'Eglise des Assemblées de Dieu nous dit à cet effet: "l'objectif premier de l'église est la socialisation de ses membres pour un mieux-être ». La socialisation des membres de l'Eglise des Assemblées de Dieu dont font partie ces femmes est le processus par lequel les autorités religieuses obtiennent qu'elles adaptent leurs comportements à leurs exigences (doctrines religieuses, valeurs et principes religieux contenus dans les Saintes Ecritures) et qu'elles tiennent compte de leurs attentes. Sur la base des valeurs qu'elles ont en commun, elles se font mutuellement confiance et travaillent ensemble à leur mieux être grâce à l'identité collective que l'Eglise a réussi à former chez ces femmes.

D'un autre côté, la palmeraie que les femmes du GFPVG ont à leur actif peut être qualifiée d'élément liant. Le palmier à huile met du temps à entrer en phase de production active et son exploitation peut s'étendre sur une période de 30 ans. Les femmes du Groupement ne peuvent aussi pour cette raison se retirer du groupement. C'est en cela que la secrétaire générale du GFPVG affirme: "je ne peux pas quitter le groupement à cause de la palmeraie ». Les femmes entendent jouir jusqu'à terme, de tous les avantages pécuniaires que peut procurer cet investissement.

Au-delà des groupements, la majorité des femmes pratique leur activité de façon libre. C'est un choix qu'elles trouvent avantageux et justifié parce

\footnotetext{
${ }^{64}$ L'Action des Ouvrières du Christ est l'organe national en charge des affaires féminine au sein de l'église des Assemblées de Dieu
} 
que selon elles, leurs activités doivent leur permettre au final de changer de statut social. Dame K.P. s’exprime en ces termes : "On a meilleur contrôle de son capital quand on travaille seul ». Elles préfèrent organiser leurs activités de façon individuelle pour ne pas risquer de voir leur gain se volatiliser et être détourné, car l'une des raisons pour lesquelles le regroupement a connu un échec dans cette localité a été les détournements de fonds. Certaines femmes trouvent aussi que les groupements sont contraignants en termes de contribution. Dame K. S. affirme : " il y a trop de cotisations dans les groupements ». En effet, La première condition pour devenir membre d'un groupement est d'abord de s'acquitter d'un droit d'adhésion. De plus des cotisations mensuelles ou circonstancielles, sont prélevées auprès des membres car les groupements ont aussi l'obligation morale d'assister leurs membres dans les évènements heureux aussi bien que malheureux. Aussi la première source de financement des groupements est la cotisation de ses membres. Les nombreuses cotisations au sein des groupements et coopératives constituent l'une des raisons qui conforte ces femmes dans les modes de pratique individuels.

D'une forme d'organisation à une autre, les femmes sont guidées par le souci de maximisation du profit. Certaines préfèrent travailler ensemble pour minimiser les efforts partant du fait qu'ensemble tout travail aussi éprouvant soit-il, peut être abattu grâce aux efforts combinés ; tandis que d'autres préfèrent exercer leurs activités de façon individuelle pour avoir un meilleur contrôle de leur gain et limiter le risque de voir leur profit se volatiliser. Le choix des activités de production, de transformation et de commercialisation de façon simultanée et le choix des modes de pratique visé par ces femmes permet de maximiser le gain économique, en minimisant les risques de perte.

Les stratégies mises en place par les femmes contribuent-elles à leur autonomisation financière?

\section{Portee socio-economique des activites des femmes}

Il est essentiellement question de montrer à quel niveau se situe la portée sociale et la portée économique des activités génératrices de revenu des femmes.

\section{Portée économique des activités féminines dans le processus d'autonomisation financière}

Les productrices et commerçantes de vivriers de la Sous-préfecture de Gomon tirent de leurs activités un gain économique qui constitue un revenu. Il s'agira pour nous de montrer grâce à la portée économique de leurs activités le niveau d'autonomie financière auxquelles elles sont parvenues. Cela consiste à mettre en évidence les besoins qu'elles parviennent à satisfaire grâce 
à la pratique de leurs activités économiques. Pour cela, il convient de faire la distinction entre les besoins solvables et les besoins non solvables. Les besoins solvables sont des besoins qu'on peut réussir à satisfaire grâce à la disponibilité des ressources économiques.

Illustrons à partir de ce tableau la proportion des femmes qui parviennent à satisfaire leurs besoins quotidiens qui sont des besoins solvables.

Tableau 3 : Proportion de femmes qui parviennent à soigner leurs familles et à se soigner elles mêmes

\section{Proportion de femmes qui parviennent à se soigner et soigner leur famille}

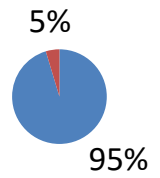

Parvient à se soigner elle et sa famille

Source : Données d'enquête (Sikensi-Gomon 2015)

Nous notons une forte proportion de femmes, soit 95\% qui parviennent à se soigner elles-mêmes et leurs familles, grâce au revenu généré par leurs activités. Leur revenu leur permet de faire face à leurs besoins en santé. Participent- elles également à faire face aux dépenses de la famille ?

Le tableau qui suit met en relief la participation des femmes aux dépenses de la famille en fonction de leur statut matrimonial.

Tableau 5 : Participation aux dépenses de la famille en fonction de la situation matrimoniale

\begin{tabular}{|c|c|c|c|c|c|c|}
\hline \multirow{2}{*}{$\begin{array}{l}\text { Participationaux } \\
\text { dépenses }\end{array}$} & \multicolumn{2}{|c|}{$\begin{array}{c}\text { Participe aux } \\
\text { dépenses }\end{array}$} & \multicolumn{2}{|c|}{$\begin{array}{c}\text { Ne participe pas } \\
\text { aux } \\
\text { dépenses }\end{array}$} & \multicolumn{2}{|r|}{ Total } \\
\hline & Eff & Fréq(\%) & Eff & Fréq(\%) & Eff & Fréq(\%) \\
\hline Mariée & 27 & 62,79 & 1 & 2,33 & 28 & 65,12 \\
\hline Célibataire & 2 & 4,65 & 2 & 4,65 & 4 & 9,30 \\
\hline Concubine & 4 & 9,30 & 0 & - & 4 & 9,30 \\
\hline Divorcée & 3 & 6,98 & 1 & 2,33 & 4 & 9,30 \\
\hline Veuve & 3 & 6,98 & 0 & - & 3 & 6,98 \\
\hline Total & 39 & 90,70 & 4 & 9,30 & 43 & 100,00 \\
\hline
\end{tabular}

Source : Données d'enquête (Sikensi-Gomon 2015)

Le pourcentage des femmes qui participent aux charges familiales s'élève à $90,70 \%$. Cette contribution est perceptible dans le règlement des factures, les besoins en nourriture et en vêtements. Presque la totalité des femmes participent aux dépenses de la famille. La plupart d'entre elles sont en couple soit 72,09\%.Grâce à leurs activités, les femmes ne dépendent plus de leurs époux. Elles cessent de recevoir et d'attendre d'eux les ressources 
financières essentielles à leurs besoins mais elles apportent une contribution à la prise en charge des dépenses familiales. Madame N., trésorière générale du GFAG, disait: "parce qu'on est dans un foyer, on ne peut pas rester oisive ». Cela veut dire que les femmes ont beaucoup plus de pression lorsqu'elles sont en couple. Elles sentent le besoin crucial d'avoir une source de revenu afin d'apporter une contribution et un soutien à l'époux dans la prise en charge familiale. Les femmes affectent plus facilement leur revenu au bien-être familial et en particulier à celui des enfants. Elles investissent plus de la moitié de leur revenu dans l'éducation de leurs enfants. Ces femmes disent vouloir être des compléments pour leurs maris et des relais quand ceux-ci ne peuvent pourvoir aux besoins économiques de la famille.

Voyons aussi, si l'activité des femmes au-delà de la satisfaction des besoins mentionnés plus haut qui sont en réalité des besoins primaires, des besoins obligatoires, leur permet de satisfaire des besoins aspirations qui correspondent à un mieux-être.

Tableau 7 : Répartition des femmes selon le niveau d'épargne et d'investissement

\begin{tabular}{|c|c|c|c|c|c|c|}
\hline \multirow{2}{*}{$\begin{array}{c}\text { Nikeau d'investissement } \\
\text { Niveau d'épargne }\end{array}$} & \multicolumn{2}{|c|}{ Réalisation } & \multicolumn{2}{c|}{ Pas de réalisation } & \multicolumn{2}{c|}{ Total } \\
\cline { 2 - 7 } & Eff & Fréq(\%) & Eff & Fréq(\%) & Eff & Fréq(\%) \\
\hline Epargne & 2 & 4,65 & 5 & 11,63 & 7 & 16,28 \\
\hline N'épargne pas & 13 & 30,23 & 23 & 53,49 & 36 & 83,72 \\
\hline Total & 15 & 34,88 & 28 & 74,42 & 43 & 100,00 \\
\hline
\end{tabular}

Source : Données d'enquête (Sikensi-Gomon 2015)

Le pourcentage des femmes qui épargnent associé à celui des femmes qui ont investi montre que près de la moitié des femmes soit 51,16\% parviennent à satisfaire des besoins aspirations. En général les femmes épargnent dans le but d'investir. Certaines d'entre elles ont déjà investi leurs épargnes dans l'acquisition de matériels de travail (machette, daba, presseuses, produits phytosanitaires, ...), et dans l'agrandissement de leurs parcelles de production. C'est le cas du GFPVG qui a développé ses activités en investissant ses épargnes dans la création d'un champ de palmier à huile de 3 hectares. Madame Y., commissaire aux comptes du GFPVG nous confie aussi : "j'ai utilisé mes épargnes pour construire ma maison. Je la voulais en brique, mais elle est en argile. Ça me donne entière satisfaction ».

Les femmes arrivent un tant soit peu à couvrir leurs besoins aspirations. Le taux de couverture s'élevant à $51,16 \%$ est encore faible mais non négligeable. Cela nous permet de dire que les femmes parviennent à satisfaire largement leurs besoins d'obligations (95\% pour la santé, 72,09\% pour les dépenses de la famille) et tendent à satisfaire les besoins aspirations $(51,16 \%)$ en vue d'un mieux-être. Les activités génératrices de revenus des femmes de la Sous-préfecture de Gomon au-delà de la portée économique ont aussi une portée sociale. 


\section{Portée sociale des activités dans le processus d'autonomisation des femmes}

La portée sociale des activités menées par les femmes peut être perçue à plusieurs niveaux. Cependant, nous allons la saisir au niveau familial et communautaire.

\section{Au niveau familial}

Les femmes recherchent à travers la pratique de leurs activités à s'émanciper, à s'affranchir de la domination masculine. Les stratégies économiques mises en place dans cette optique visent à acquérir un statut économique et social décent au sein de la famille et de la communauté. Le peuple Abidji est un peuple patrilinéaire. Cette forme d'héritage met l'homme sur un piédestal. Cet état de fait lui accorde beaucoup plus de privilèges, de droits et accentue le statut précaire de la femme en la reléguant au second plan. Le chef du village de Gomon affirme : "l'homme Abidji est paresseux. C'est la femme qui doit tout faire ». Ces propos traduisent le fait que la femme soit celle qui doit faire "fonctionner", faire "vivre" la famille. Elle doit remplir efficacement ses tâches pour que subsiste la famille. Les femmes doivent travailler plus que l'homme pour se distinguer et pour avoir la considération de celui-ci et de la communauté parce que le système de valeur et d'organisation traditionnelle favorise une vision phallocratique. A travers leurs activités économiques les femmes veulent revaloriser leur statut social. Leurs activités leur permettent d'assurer les besoins de la famille et leur confèrent un certain pouvoir vis-à-vis de l'homme. Madame K., présidente du GFAG témoigne en ce sens: " j'assure tous les besoins de la famille. Mon mari n'est là que pour la forme ". L'homme est en voie de perdre sa position éminente dans le foyer. L'époux de cette dame citée plus haut nous confie : «ma femme est le véritable soutien de la famille. Elle est une aide appréciable ». Il complète pour dire : " il faut garder une telle femme chez soi ». Les femmes grâce à leurs activités sécurisent aussi leur place dans le foyer. Le témoignage de madame N., secrétaire générale du GFAG nous le confirme aussi : " mon mari m'avait répudiée pour une autre. Cette dernière était paresseuse. Mon travail et le fruit de mes activités m'ont ramené dans mon foyer 》.

$\mathrm{Au}$ vu de ce qui précède nous pouvons dire que les activités économiques des femmes de Gomon ont un impact positif. Elles leurs ont permis de revaloriser leur statut d'épouse. En tant que partenaires privilégiées, les femmes garantissent leur place dans le foyer et ont le respect et l'admiration de leurs époux. Cela s'est fait grâce aux fruits de leurs activités économiques qui leur confèrent une autonomie financière. Les activités des femmes de la sous-préfecture de Gomon ont aussi une portée sociale perceptible au niveau communautaire. 


\section{Au niveau communautaire}

La portée sociale des activités des femmes de la sous-préfecture de Gomon au niveau communautaire est perçue à travers leur capacité à participer à la vie sociale. Grâce à leurs activités, elles participent aux cotisations lors des évènements tels que les funérailles, les mariages, les naissances, les cas de maladie...Elles interviennent mieux dans la construction des valeurs sociales que sont l'entraide et la solidarité grâce aux revenus dont elles disposent désormais. Les femmes recherchent aussi la reconnaissance et le respect de la communauté et l'intégration sociale. Les propos de Madame B., présidente du GFPVG, l'attestent bien : «il ne faut pas rester sans rien faire sinon on n'a pas de considération pour toi. Je recherche le respect de la communauté ». Elle termine en disant : "ma satisfaction est entière parce que je me sens respectée mais aussi intégrée à la vie sociale ». Les femmes à travers les activités génératrices de revenus veulent être socialement reconnues et intégrées à la vie sociale. Elles veulent asseoir leur leadership en ne restant plus en marge de la vie sociale mais en devenant des acteurs de développement. Elles mènent des actions sociales en faveur de leur communauté grâce aux revenus de leurs activités. C'est le cas des femmes réunies dans le GFPVG qui ont refait la peinture de l'église des Assemblées de Dieu de Gomon à laquelle elles appartiennent. Les activités génératrices de revenu des femmes de la Sous-préfecture de Gomon leurs valent le respect de la communauté toute entière, d'être socialement reconnues et d'être intégrées à la vie sociale.

\section{Discussion}

\section{Mise en perspective de la femme rurale stratège face au défi de son autonomisation}

C'est en travaillant par le passé aux côtés de l'homme en tant que main d'œuvre que les femmes participent au développement des cultures d'exportation sans avoir de revenu. La nécessité d'avoir un revenu, favorisé par un souci d'indépendance économique vis-à-vis de l'homme dont le revenu est affecté par la crise économique (Bulteau, 1992) va donner naissance aux activités rurales féminines. Les activités mises sur pied par les femmes doivent leur générer du profit afin de se rendre autonome et de s'émanciper. Le comportement des femmes dans la pratique de leurs activités relèvent d'une stratégie. Leurs actions inscrites dans une perspective financière émanent d'un processus d'allocation rationnelle de ressources rares à usage alternatif par lequel les femmes cherchent à obtenir le maximum de moyens dont elles disposent pour satisfaire les objectifs de leur travail. Pour ce faire, en fonction de leur perception de la réalité, les femmes choisissent selon les risques et les avantages inhérents, les activités à mener. A savoir la production, la transformation et la commercialisation du vivrier. Le choix du type de vivrier 
produit est très sélectif, la logique étant de minimiser les risques de perte afin de maximiser le gain économique. Une gestion du risque et de l'incertitude transparait dans la conduite des activités de ces femmes. Par un calcul du gain et de la perte, elles mesurent les risques liées à toute décision dans l'exercice de leurs activités. Elles prouvent qu'elles sont des êtres conscients qui décident à l'aide de calculs rationnels, de la ligne d'action qui parmi d'autres est la plus susceptible de maximiser leurs récompenses globales (Rule, 2002). Selon l'auteur, on peut aussi supposer que les individus supputent différentes lignes d'action et modifient leur conduite lorsque de nouvelles stratégies offrent de meilleures chances de donner les résultats désirés.

Dorénavant, les femmes de par leurs activités sortent du mode de production paysan précolonial, inséré dans un système de solidarité, d'échanges et d'obligations morales fondé sur les liens affectifs de la parenté ...fournit à tous un filet de sécurité et une assurance contre les risques pour emprunter la voie de l'entrepreneuriat et de la création de devise (Sardan, 2013). Elles se sont accommodées à la de recherche de profit. Sardan (op. cit.) soutient cette thèse lorsqu'il confirme que: les logiques marchandes, la recherche du profit, l'usage des institutions modernes leurs sont devenues largement familiers. En effet, le modèle ivoirien s'est construit largement en dehors de toute référence à l'agriculture vivrière. Ce secteur était au départ perçu comme secondaire, voire archaïque, voué à l'autosuffisance, en marge de l'économie marchande (Chaléard, 1994). Les cultures vivrières sont devenues progressivement des cultures marchandes en raison d'une forte croissance démographique qui a provoqué un essor considérable des productions destinées aux villes. Cette mutation de cultures de subsistance en cultures marchandes a permis à la femme de créer du profit, de s'affirmer et de revaloriser son statut social. Les femmes ont réalisé que de nouvelles opportunités pouvant les sortir de la précarité leurs sont offertes. Elles produisent donc, transforment et font la commercialisation des cultures vivrières pour atteindre un but qui est de devenir autonome. Leur logique, est de maximiser le gain économique afin de lutter efficacement contre la pauvreté.

\section{Logiques sociales de légitimation des activités rurales féminines}

Des logiques sociales conduisent aussi les femmes à s'investir dans les activités socio-économiques. Les femmes recherchent à travers la dynamisation de leurs activités à être socialement reconnues. De l'avis unanime des experts en matière de pauvreté, l'un des moteurs principaux permettant de réduire la pauvreté et d'assurer la croissance économique est le renforcement du statut des femmes. L'organisation sociale villageoise a toujours relégué la femme au second plan, la privant de droit à la terre, reconnue comme source primordiale de richesse, de statut social et de pouvoir 
(Affessi, 2014). En milieu rural, l'intégration ou l'exclusion sociale dépendent souvent du statut d'une personne en matière de droit foncier. Les femmes à travers la pratique de leurs activités économiques, revendiquent leur intégration dans le tissu social. La revalorisation de leur statut et le renforcement de celui-ci est d'abord le combat pour lequel elles s'engagent à travers la pratique de leurs activités économiques. Ces activités ne s'inscrivent pas seulement dans une logique économique. Derrière cette logique économique se cache une logique sociale. C'est en effet, ce que (Toh, 2000) atteste à travers la recherche du sens et des significations profondes de l'activité économique des femmes. Pour lui celles-ci s'adonnent aussi aux activités économiques pour des raisons non matérielles à savoir la recherche d'une réussite sociale. Les femmes de la Sous-préfecture de Gomon recherchent surtout une estime et une considération sociale. (Clelland, 1961) n'est pas de cet avis car pour lui, les motivations qui peuvent inciter des acteurs à s'investir dans des activités économiques tiennent lieu d'un besoin d'accomplissement; un désir de bien faire, non pour obtenir considération sociale ou prestige mais pour atteindre un sentiment intérieur d'accomplissement personnel. En toute réalité, on ne peut se limiter à la dimension sociale ou économique pour légitimer les actions humaines. Ainsi, Maslow (1943) propose une approche différente des motivations, à l'origine des conduites humaines. Des raisons psychosociologiques pourraient conduire les femmes de Gomon à s'impliquer dans leurs activités. Il distingue cinq grandes catégories de besoins humains. Au niveau 1 de la pyramide il situe les besoins physiologiques, qui correspondent à des besoins de nourriture, d'habillement...que nous avons assimilé aux besoins obligations. Les besoins de sécurités, au niveau 2 de la pyramide concernent la sécurité physique, mais aussi morale, psychologique, affective...Le besoin d'appartenance apparait au niveau 3 de la pyramide et relève de la dimension sociale de l'individu qui a besoin de se sentir accepté par le groupe dans lequel il vit. L'individu souhaite être reconnue en tant qu'entité propre, au sein du groupe dans lequel il appartient. Les femmes de Gomon se sont longtemps senties exclus de la société ; elles n'ont pas toujours été intégrées à la vie sociale. Le besoin d'estime prolonge le besoin d'appartenance. L'individu souhaite être reconnu en tant qu'entité propre au sein des groupes auxquels il appartient. Les femmes ont besoin de se sentir estimer, considérer et valoriser dans leur société d'appartenance. Maslow (1943) place au sommet de sa pyramide, le besoin d'accomplissement qui vise à sortir d'une condition purement matérielle pour atteindre l'épanouissement. C'est également le besoin de participer fût-ce modestement à l'amélioration du monde. Il pense toutefois que le passage d'un niveau à l'autre ne pourrait s'effectuer que si le besoin du niveau inférieur est satisfait (Maslow, 1943). A ce stade les femmes deviennent des agents de développement social et participent à l'amélioration de leur environnement 
social. Sur le plan conjugal, l'on enregistre une mutation du rôle social. Des femmes jouent le rôle de chef de famille en pourvoyant aux ressources indispensables à la prise en charge de la famille. Elles montrent qu'elles sont fortes, braves, capables de se prendre en charge, mais bien plus, de prendre en charge leur famille et contribuer au mieux-être de leur communauté. Cela pourrait justifier le besoin d'accomplissement selon Maslow (1943).

\section{Conclusion}

L'étude portant sur les activités des femmes en milieu rural dans la problématique de l'autonomisation nous a permis de mettre en évidence les stratégies mises en œuvre par les femmes pour lutter contre la pauvreté. Les femmes de la Sous-préfecture de Gomon se comportent en véritables agents économiques (homoéconomicus). Elles sont présentées comme des êtres conscients et rationnels qui opèrent des choix en fonction de leurs intérêts et en tenant compte du contexte dans lequel elles se trouvent. Leur choix concernant les activités de production, de transformation et de commercialisation du vivrier menée simultanément, vise à minimiser les risques de perte et à maximiser le gain économique. Cette gestion du risque transparait aussi dans les modes de pratique de leurs activités. En effet les femmes trouvent un intérêt substantiel à se regrouper ou à exercer leurs activités de façon individuelle. Grâce à leurs stratégies, les femmes parviennent à satisfaire leurs besoins obligations (95\% pour la santé, $72,09 \%$ pour les dépenses de la famille) et tendent à satisfaire des besoins aspirations soit $51,16 \%$ pour l'épargne et l'investissement. Les activités économiques des femmes de la Sous-préfecture de Gomon leur permettent d'être autonomes financièrement grâce à la maximisation de leur profit et leur valent d'être socialement reconnues et intégrées à la vie sociale

\section{References:}

1. Affessi, A. S. (2014). «Encadrement des paysans et développement rural : l'exemple de l'ANADER dans les milieux ruraux d'Adzopé (Côte d'Ivoire) » in Journal Africain de Communication Scientifique et Technologique, $\mathrm{n}^{\circ} 25$. Abidjan : IPNETP, PP.3223-3239

2. Baha Bi, Y.D.(2001) «Genre et développement : analyse sectorielle de la situation socio-économique, politique et Juridique de la femme en Côte d'Ivoire »,Kasa Bya Kasa ,Revue Ivoirienne d'Anthropologie et de Sociologie $\mathrm{n}^{\circ} 2$, p.50-65.

3. Bulteau, P. (1992). compte rendu de la mission “intégration planteurs agro-industrie“(projet UNICAFE qui s'est déroulé du 04 Novembre au 07 Décembre 1991), CIRAD-DSA Montpellier

4. Chaleard, J.L.(1994) "' L'essor du vivrier marchand: un contre modèle aux marges du modèle ivoirien' ' communication au colloque 
international Gidis-CI, ORSTOM, crise, ajustements et recompositions en Côte d'Ivoire : la remise en cause d'un modèle. Abidjan, le 28 novembre -2 décembre 1994.

5. Droy, I (1990) Femmes et développement rural,Paris, Karthala,186p.

6. Koné, M.(2003). «Les femmes et l'accès à la terre en milieu rural ivoirien ", Regard sur le foncier rural en Côte d'Ivoire p.5180Abidjan, INADES/NEI/CERAP

7. Kouadio,N.P.(1993). L'activité économique des vendeuses de produits vivriers du grand marché d'Adjamé: élément pour une analyse Wéberienne, mémoire de maitrise, IES, Université de cocody, Abidjan

8. Maslow, A .(1943). "A théory of human motivation " in Psychological review, 50, 370-396.

9. Mcclelland. (1961).Achieving society, Van Nostrand.

10. Olivier de Sardan, J.P. (1993): Anthropologie et développement : Essai en socio-anthropologie du changement social APAD- Karthala, $203 \mathrm{p}$

11. Raffaillac, J.P. (1997). Le manioc : quelles priorités de recherche pour améliorer la production en relation avec la transformation et la commercialisation? in CIRAD, archives de documents de la FAO.

12. Rule, J.B. (2002). «Les leçons du choix rationnel ».Sociologie et sociétés, vol 34, $\mathrm{n}^{\circ} 1, \quad$ p.51à $66 . \quad \mathrm{En}$ ligne, http://id.erudit.org/iderudit/009745 ar.

13. Savane, M.A. (1986). Femmes et développement en Afrique de l'ouest : incidences des transformations socio-économiques sur le rôle et le statut des femmes, UNRISD, Génève, 207p.

14. Toh, A. (2000) Femmes et développement rural : étude des activités socioéconomiques des femmes rurales de la sous préfecture de Boguédia, dans le département d'Issia, mémoire de maîtrise, IES, Université de Cocody, Abidjan.

15. Vanga, A.F.(2012). «Genre et production agricole dans les coopératives du Nord de la Côte d'Ivoire ", European Scientific Journal, ${ }^{\circ} 30$, p. 174-187

16. Organisation des Nations Unies pour l'alimentation et l'agriculture (2010). Que fait la FAO? Justice sociale:Égalité des droits entre hommes et femmes In Organisation des Nations Unies pour l'alimentation et l'agriculture. En ligne, http://www.fao.org/kids/fr/equalrights.html 